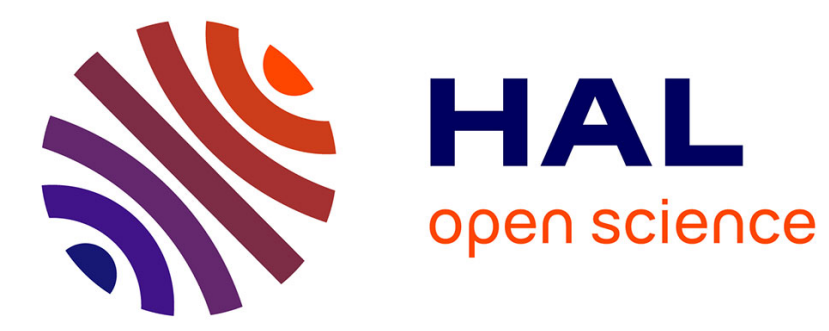

\title{
POINCARÉ ET LE PROBLÈME DE L'ESPRIT
}

Gabriella Crocco

\section{To cite this version:}

Gabriella Crocco. POINCARÉ ET LE PROBLÈME DE L'ESPRIT. Revue de Métaphysique et de Morale, 2016, La naissance de l'épistémologie française histoire et perspectives, 90, pp.209-224. 10.3917/rmm.162.0209 . hal-03388976

\section{HAL Id: hal-03388976 https://hal.science/hal-03388976}

Submitted on 20 Oct 2021

HAL is a multi-disciplinary open access archive for the deposit and dissemination of scientific research documents, whether they are published or not. The documents may come from teaching and research institutions in France or abroad, or from public or private research centers.
L'archive ouverte pluridisciplinaire HAL, est destinée au dépôt et à la diffusion de documents scientifiques de niveau recherche, publiés ou non, émanant des établissements d'enseignement et de recherche français ou étrangers, des laboratoires publics ou privés. 


\title{
POINCARÉ ET LE PROBLÈME DE L'ESPRIT
}

GABRIELLA CROCCO

Aix-Marseille Université CEPERC UMR 7304

\begin{abstract}
RESUME - La conception du continu de H. Poincaré, telle qu'elle est exposée dans le chapitre 2 de La Science et l'hypothèse est le résultat du croisement de considérations mathématiques, philosophiques et physiques. Elle est également subordonnée à une théorie des facultés qui renouvelle l'épistémologie kantienne tout en la réinterprétant dans le débat de l'époque. Le but de cet article est double : montrer l'articulation interne de cette conception du continu de Poincaré avec les doctrines mathématiques et physiques qui ont été les siennes et montrer son originalité dans le débat épistémologique de l'époque.
\end{abstract}

ABSTRACT - Poincaré's conception of the continuum, such as is presented the second chapter of Science and Hypothesis is the result of convergence of mathematical, physical and philosophical considerations. It is commanded by a theory of faculties that renew with the Kantian epistemology and reinterpret it in the contemporary debate. This paper has a double aim: to show the internal connexion of Poincaré's solution to the problem of the continuum with his mathematical and physical conviction and to strength its originality in the epistemological panorama of its time.

\section{LA RÉFLEXION ÉPISTÉMOLOGIQUE DE POINCARÉ ${ }^{1}$}

Y a-t-il un problème de l'esprit chez Poincaré ? Peut-on trouver dans son travail épistémologique une réflexion articulée qui aboutit au constat d'une dualité irréductible et fondatrice de notre esprit et qui aurait comme point de départ le rejet de l'idée que notre expérience du monde soit simple, uniforme et directe, et qu'elle puisse nous donner un accès à la réalité que la science n'aurait qu'à étendre ?

Je donnerai dans cet article une réponse positive à ces questions : Poincaré participe sans aucun doute à la formulation et à l'analyse de ce qui a été appelé par Frédéric Worms le problème de l'esprit « du moment $1900^{2}$ ». Il me semble en effet indéniable que, sous fond de réflexion renouvelé du kantisme et de remise en question de l'apriorisme, Poincaré pose à nouveaux frais le problème du rapport de l'espace au temps dans la constitution de l'expérience et qu'il en tire plusieurs conclusions quant à la puissance et aux limites de l'esprit.

Néanmoins, j'essayerais de montrer, contrairement à ce qu'avance Worms, que ce ne sont pas les problèmes posés par les géométries non-euclidiennes qui motivent et animent les interventions épistémologiques de Poincaré. Si l'on peut affirmer que les géométries noneuclidiennes ont été chez lui la source de l'idée « d'un esprit qui à la fois s'intercale entre le réel et en même temps nous y insère autrement que pour seulement y agir, pour le connaitre, pour en connaître l'ordre et la beauté propres ${ }^{3} »$, il reste toutefois encore à comprendre de manière précise la nature et la valeur de cette connaissance à laquelle aboutit ce double mouvement de voilement et d'insertion et ce n'est pas la géométrie mais la mécanique et la notion de loi physique qui sont le terrain réel sur lequel se développe sa réflexion

\footnotetext{
${ }^{1}$ Les réflexions consignées dans cet article, écrit à l'occasion de ce numéro spécial de la RMM sur le début de l'épistémologie en France, ont en partie muris pendant les trois ans de discussions du groupe de travail en philosophie des mathématiques du CEPERC d'Aix-Marseille auquel ont participé en particulier Alain Michel, Eric Audureau et Julien Bernard. Le but de ce travail devait être la rédaction d'une édition annotée et commentée de La Science et l'hypothèse. Bien que nous espérons encore que la publication de ce travail en grand partie accompli verra enfin le jour, j'ai pensé pouvoir librement utiliser certaines des parties de ce que j'avais moimême rédigé pour ce travail. Je dois par contre à Alain Michel les informations contenues dans la note 7 sur Brunetière et à Eric Audureau l'idée de la nécessité d'une comparaison sérieuse entre Poincaré et Duhem, trop souvent associés sous l'étiquette vide de «conventionnalistes ».

${ }^{2}$ F. Worms La Philosophie en France au XX siècle. Moments Gallimard 2009

${ }^{3}$ Idem page 87
} 
Poincaré s'exprime de manière on ne peut plus explicite à ce propos dans l'introduction de la Valeur de la Science, rédigée en 1905. Ayant distingué la vérité scientifique de la vérité morale, tout en soulignant que les raisons qui nous les font aimer ou redouter sont les mêmes, il s'interroge sur les conditions de possibilité de la première et il se demande :

L'analyse mathématique, dont l'étude de ces cadres vides [le temps et l'espace, les cadres dans lesquels la nature nous paraît enfermée] est l'objet principal, n'est-elle donc qu'un vain jeu de l'esprit? Elle ne peut donner au physicien qu'un langage commode ; n'est-ce pas là un médiocre service, dont on aurait pu se passer à la rigueur; et même n'est-il pas à craindre que ce langage artificiel ne soit un voile interposé entre la réalité et l'œil du physicien? Loin de là, sans ce langage, la plupart des analogies intimes des choses nous seraient demeurées à jamais inconnues; et nous aurions toujours ignoré l'harmonie interne du monde, qui est, nous le verrons, la seule véritable réalité objective ${ }^{4}$.

Et après avoir affirmé que la meilleure expression de cette harmonie c'est la loi physique dont les lois de la mécanique céleste ont été le modèle, il ajoute :

Cette harmonie que l'intelligence humaine croit découvrir dans la nature, existe-t-elle en dehors de cette intelligence ? Non, sans doute, une réalité complètement indépendante de l'esprit qui la conçoit, la voit ou la sent, c'est une impossibilité. Un monde si extérieur que cela, si même il existait, nous serait à jamais inaccessible. Mais ce que nous appelons la réalité objective, c'est, en dernière analyse, ce qui est commun à plusieurs êtres pensants, et pourrait être commun à tous; cette partie commune, nous le verrons, ce ne peut être que l'harmonie exprimée par les lois mathématiques ${ }^{5}$.

Par le biais de l'appel à l'harmonie des lois de la physique mathématique, thème récurrent depuis Cournot sous la plume des scientifiques de l'époque, ces lignes indiquent clairement les éléments du programme épistémologique de Poincaré : rendre à la loi physique (identifiée à la loi scientifique tout court) ses droits présuppose d'expliquer comment ce qui pourrait s'interposer comme un voile entre nous et la réalité, c'est-à-dire le langage, peut prendre dans les mathématique une forme capable au contraire d'épouser de près ce qui est constitutif de notre expérience. En d'autres termes et comme on le verra par la suite, il faut montrer que les limites même de notre connaissance, sont, en même temps, les conditions de possibilité. A ceux qui ne redoutent pas la vérité scientifique et qui savent reconnaître que la connaissance objective doit être entendue comme ce qui peut être commun à tous les esprits, reste donc à analyser de près les objets principaux d'études des mathématiques, l'espace et le temps, et de décrire comment il permettront de déceler ces analogies intimes des choses. C'est alors par l'analyse des rapports entre mathématiques et expérience que se révèle cette dualité fondamentale et constitutive de notre esprit, qui invite à dissocier temps et espace, ou du moins à reconnaître que « le temps est antérieur logiquement à l'espace ${ }^{6}$ » avec toutes les conséquences que ce constat comporte pour Poincaré pour l'analyse des conditions de possibilité de la loi physique. Ce faisant, Poincaré veut simultanément montrer que les lois de la nature ne sont pas contingentes, qu'elles ne sont pas artificielles et qu'une analyse attentive de ce qui est commun à l'expérience et au langage des mathématiques révèle leur vérité. Ainsi l'analyse des mathématiques est un élément essentiel dont le physicien comme le philosophe peuvent tirer profit pour préciser les transformations qu'il faut accomplir pour rétablir la confiance en la valeur de la science et en sa vérité, à une époque où certains dénoncent sa banqueroute ${ }^{7}$.

\footnotetext{
${ }^{4}$ H. Poincaré, La Valeur de la science, Flammarion 1970 (première édition 1905) p, 22.

5 Idem page 23

${ }^{6}$ Idem, chapitre IV, section : L'esprit et l'espace, p.98.

${ }^{7}$ L'expression " banqueroute de la science" a été utilisée pour la première fois en 1895 par Ferdinand Brunetière dans un ouvrage publié à Paris, et intitulé La science et la religion. Mais c'était pour protester contre
} 
Si l'on restitue à la réflexion sur la crise de la mécanique, laquelle avait constitué l'idéal même de la rationalité au XVIII ${ }^{\mathrm{e}}$ siècle, le rôle central qu'elle a dans la pensée de Poincaré, non seulement la cohérence et l'unité de sa réflexion apparaissent avec netteté, mais se dessine de plus avec force son opposition à Pierre Duhem, jamais ouvertement mis en cause mais que bien des aspects opposent à Poincaré, à commencer par la vision des rapports entre science et philosophie.

Telles sont les thèses que je voudrais défendre. Il me reste à décrire le cheminement de mon argumentation. D'abord, je rappellerai certains aspects du contexte des interventions de Poincaré dans la Revue de métaphysique et de morale (dorénavant RMM) entre 1893 et 1905, qui ont donné la matière de ses deux premiers ouvrages épistémologiques La Science et l'hypothèse de 1902 et La Valeur de la science de 1905. Je me limiterai, à quelques exceptions près, aux contributions de Poincaré durant cette période car elles sont les plus directement impliquées dans la naissance de l'épistémologie française.

Ensuite les deux sections suivantes illustreront, sur le cas spécifique de l'analyse mathématique, physique et philosophique du continu, le rôle que Poincaré assigne, par l'analyse du langage mathématique dans la connaissance de la nature, à l'enquête épistémologique.

\section{POINCARÉ, BOUTROUX ET LA RMM}

Laurent Rollet rappelle que lors du lancement de la $R M M$, Xavier Léon et Élie Halévy souhaitaient bénéficier de la collaboration de savants renommés et il semble que Bergson leur conseilla de demander un article à Boutroux et Poincaré. Un lettre de Léon à Halévy datée d'octobre 1892 en témoigne :

J'ai vu Bergson et j'ai vu Couturat. Bergson se propose pour le second numéro mais ne veut pas entendre parler du $\mathrm{I}^{\mathrm{er}}$ pour des raisons de temps et des raisons de prudence sémitique. Couturat m'a rapporté les livres en déclarant qu'il se sentait tout à fait incapable de discuter avec Renouvier, il remet la discussion à un an. Voilà où j'en suis et je commence à sentir la lourdeur de ma mission. Bergson est d'ailleurs on ne peut mieux disposé et plus aimable : il travaillera pour la Revue à mettre au point quelque théorie de la perception extérieure qui paraîtra un jour en volume et dont sa thèse n'est qu'un extrait. Mais malgré toute ma vive insistance je n'ai pu le décider à me donner rien de ferme pour le mois de décembre: des promesses et des encouragements c'est beaucoup, mais c'est trop peu. Il m'engage à voir Boutroux pour lui arracher un article ainsi qu'à Poincaré. Il voudrait pour lancer la Revue les trois noms de Ravaisson, Boutroux (essentiel dit-il comme porte-parole puisqu'il est notre maître à tous, et il m'a promis d'aller le voir à ce sujet) Poincaré - je ne demande pas mieux, mais encore faut-il les avoir. ${ }^{8}$

Je voudrais m'arrêter ici sur deux point que cette citation soulève. Le premier concerne le rôle reconnu à Boutroux, rôle qui invite à considérer attentivement sa contribution à la formulation du problème de l'esprit. La seconde concerne Poincaré et la manière dans laquelle ses nombreuses interventions dans la revue révèlent sa dette de même temps que sa distance à 1 'égard de Boutroux.

En quel sens, donc et en premier lieu, Boutroux peut-il être considéré comme le père spirituel du cercle de la $R M M$ à laquelle Poincaré est invité à contribuer ?

l'exagération qu'elle implique. Il a tenu à s'en expliquer l'année suivante dans une conférence intitulée $L a$ renaissance de l'idéalisme (prononcée le 2 février 1896, recueillie ensuite dans Discours de combats, 1900-1907, première série). S'il y maintient que les sciences avaient connu "des faillites partielles", le contexte montre que ces dernières doivent être entendues relativement aux promesses initiales. Ces dernières sont interprétées par Brunetière comme des engagements pris par les sciences, et par leurs représentants, en particulier Descartes dans le Discours de la Méthode, Condorcet dans l'Esquisse d'un tableau des progrès de l'esprit humain", Renan dans L'Avenir de la science, "pour ne rien dire de M. Berthelot".

${ }^{8}$ Laurent Rollet, Henri Poincaré, des mathématiques à la philosophie. Thèse de Doctorat, Université de Nancy II, page 163 . 
On ne peut trouver de réponse plus exemplaire à cette question que celle publiée dans la même $R M M$ en 1922 par Léon Brunschvicg9 ${ }^{9}$. D'abord, dit Brunschvicg en prenant une orientation qui poursuivait la réflexion de Jules Lachelier en France et de Eduard Zeller et Helmholtz en Allemagne, Boutroux s'insère dans ce mouvement original de retour à Kant conçu comme un antidote à la fois au positivisme et à l'Hégélisme de la Naturphilosophie, tous deux ayant en commun, malgré leurs différences, l'idée d'un déterminisme qui ferait suivre à la nature et à l'histoire une nécessité interne dominant tout aspect individuel et contingent des actions et des événements. En ce sens Boutroux est surtout le rénovateur des études d'histoire de la philosophie en France, opposant au déterminisme historiciste des hégéliens l'idée de la libre création individuelle agissant comme une puissance capable de rompre plus au moins définitivement le fil de la continuité historique. Contre le positivisme, Boutroux prône la fin d'une conception mécaniste de la science, où la nécessité métaphysique des lois est soutenue par le recours à une logique qui trahit la science au lieu de la servir puisqu'elle prétend fonder sur les exigences a priori de la raison ou sur le caractère analytique de la démonstration scientifique la nécessité de droit des lois scientifiques. Cet intérêt vers la réalité et la science détermine la fin des cloisons étanches entre science et philosophie qui avaient dominé les trois premiers quarts du $\mathrm{XIX}^{\mathrm{e}}$ et il ouvre la possibilité d'un dialogue qui prélude au renouvellement de la «nouvelle épistémologie scientifique ». Brunschvicg n'a aucun doute sur le rôle de l'ouvrage de Boutroux publié en 1874 sur cette dernière:

La thèse De la Contingence des lois de la nature définit le moment où la critique du savoir scientifique prend conscience de soi, procédant à l'examen de la science pour la science, sans référence à un parti-pris métaphysique, en particulier sans le postulat de l'apriorisme que l'autorité de Kant paraissait avoir joint par une association indissoluble à l'attitude proprement critique. De quelle importance a été l'événement, le recul de presque un demi-siècle permet de le mesurer. La thèse prélude à ce renouvellement de la philosophie scientifique, auquel les savants français ont pris une part si active, qui se trouvera consacré dans les travaux de Henri Poincaré et de Pierre Duhem. Si la nécessité du mouvement hégélien régissait le monde, si les faits devaient apparaître suivant l'ordre de l'enchaînement logique, la philosophie de Boutroux serait issue d'une réflexion sur ces travaux. En réalité elle leur est antérieure. Lorsque Boutroux soutint sa thèse sur la Contingence en 1874, Henri Poincaré, auquel il devait être lié plus tard par une union étroite de parenté et d'affection, était élève à l'école polytechnique et Duhem avait $13 \mathrm{ans}{ }^{10}$.

Si la Contingence des lois de la nature est un prélude à l'œuvre de Poincaré, il s'agit toutefois d'un prélude qui pose un défi, plutôt que d'annoncer simplement des thèmes de réflexion, un défi même plus grand que celui incarné par Le Roy et que Poincaré n'a pas de mal à relever. En effet, Poincaré bien qu'épousant sans hésitation (et avant sa polémique avec le logicisme) la méfiance de Boutroux envers la logique, rejette l'affirmation de la contingence des lois de la nature. Un article tardif et à bien des aspects étonnant «L'évolution des Lois ${ }^{11}$ », explicite ce qui a probablement été le cadre des discussions entre les deux beaux-frères :

M. Boutroux, dans ses travaux sur la contingence des lois de la nature, s'est demandé si les lois naturelles ne sont pas susceptibles de changer et si, alors que le monde évolue continuellement, les lois elles mêmes c'est-à-dire les règles suivant lesquelles se fait cette évolution, seront seules exemptes de toute variation. Une pareille conception n'a aucune chance d'être jamais adoptée par les savants ; au sens où ils l'entendraient, ils ne sauraient y adhérer sans nier la légitimité et la possibilité de la Science. Mais le philosophe conserve le droit de se poser la question, d'envisager les diverses solutions qu'elle comporte, d'en examiner les conséquences et de chercher à les concilier avec les légitime exigences des savants ${ }^{12}$.

Le dialogue entre le philosophe et le savant, se noue donc autour de la tentative de conciliation entre les légitimes exigences du savant et l'attitude critique du philosophe.

\footnotetext{
${ }^{9}$ L. Brunschvicg, « La Philosophie d'Emile Boutroux », RMM (1922) pp.261-283. Il sera cité à partir de la réédition de 1954 L. Brunschvicg, Ecrits philosophiques, vol II, Puf, 211- 31.

${ }^{10}$ Idem, p.220.

${ }^{11}$ Publié d'abord en 1911 dans la revue Scientia (Rivista di scienza)(1911) t.9, pp275-92 et ensuite reproposé posthume dans les Dernières pensées (pp. 5-32)

12 Idem, p.5
} 
Or, on l'a vu, la légitimité de la Science et sa valeur s'appuient, pour Poincaré, sur le bien fondé de la loi. Comment alors en rejetant toute forme d'apriorisme, pourrait-on s'assurer de sa possibilité, c'est-à-dire s'assurer de l'adhérence et de la communauté de structure entre le langage des mathématiques et notre expérience que ce langage traduit? Comment se peut-il que nous trouvions, par le biais de ce langage mathématique, le moyen de découvrir les analogies cachées entre les choses, si aucune structure a priori, aucun des cadres dans lesquels nous pensons les phénomènes, ne garantit le fondement commun, dans tous les esprit, de ces mêmes phénomènes.

Que l'espace et le temps ne soient pas a priori, Poincaré le reconnait sans problème. Mais alors, si les conditions mêmes de la construction des objets de l'expérience ne garantissent plus leur intersubjectivité, l'accord commun des esprits qui est gage de vérité, comment la loi scientifique est-elle possible?

La question a du être d'autant plus pressante que Poincaré entendait l'analyse que Duhem construisait en même temps. En 1892, Duhem - Poincaré avait participé au jury de sa thèse- publie dans la Revue des questions Scientifiques ses "Quelques réflexions au sujet des théories physiques ». L'interprétation de la théorie physique qu'il construit, et qu'il expliquera avec beaucoup plus de détails dans ses écrits ultérieures, y est déjà dessinée : le langage mathématique permet de traduire les données de l'expérience en termes de grandeurs mathématiques et dans cette traduction il y a toute la trahison qui est à l'origine des controverses métaphysiques. Ce sont ces controverses qui font qu'une théorie physique ne s'érige que pour s'écrouler. En effet, d'une part les définitions conventionnelles des grandeurs physiques que permettent les mathématiques sont arbitraires. D'autre part il n'y a aucune univocité entre les valeurs des grandeurs physiques que les équations différentielles ${ }^{13}$ nous permettent de déduire et les aspects de l'expérience que nous allons identifier à ces grandeurs. À cause de cette plurivocité des rapports entre langage mathématique et expérience et des tendances ontologiques intrinsèques au passage des données de la sensation à la grandeur mathématique, aucune expérience cruciale n'est envisageable.

De surcroit, la conception des mathématiques propre à Duhem, comme il le reconnaîtra explicitement dans son article de $1912^{14}$, est tout à fait opposée à celle de Poincaré. Le raisonnement mathématique peut être ramené à la syllogistique et que se soit dans l'arithmétique, dans l'algèbre ou dans l'analyse le seul aspect essentiel du raisonnement mathématique est la construction de combinaisons symboliques de plus en plus complexes.

Le mathématicien Poincaré ne peut à l'évidence accepter cette caractérisation du rôle des mathématiques dans l'expérience et la $R M M$ devient pour lui le lieu de ce dialogue serré avec la philosophie et la physique, où les cibles polémiques visées au fur et à mesure ne font que lui offrir l'occasion de tisser la trame de sa solution au problème de la loi, que les ouvrages épistémologiques de 1902, 1905 explicitent dans leur structure. Il ne faudrait pas en effet sous-estimer que dans sa collaboration avec la $R M M$ dès son premier numéro, Poincaré indique de manière directe l'enjeu de ses interventions : la nature des lois physiques. Les deux premiers articles ${ }^{15}$ portent en effet respectivement sur le continu et la mécanique et posent de manière claire, bien que par des biais et des formats différents, la question des rapports entre mathématiques et expérience. Le premier article, ensuite intégré avec des modifications dans le second chapitre de La Science et l'hypothèse, discute en effet le

\footnotetext{
13 Duhem ne parle pas d'équations différentielles mais des symboles mathématiques figurant dans le langage algébrique de la physique mathématisée.

${ }^{14}$ P. Duhem « La nature du raisonnement mathématique » Revue de philosophie t.21, p. 531-43. Il est opportun de rappeler que Abel Rey publiera en 1904 dans la $R M M$ une analyse détaillée de l'œuvre de Duhem et que au Congrès mondial de Physique de 1900, Hadamard avait accusé Poincaré d'emprunter à Duhem, sans le mentionner, ses considérations sur le caractère révisable des théories physiques.

15 « Le continu mathématique », RMM (1893) vol.1, pp. 26-34; « Mécanisme et expérience », idem, pp.534-37.
} 
problème de la mathématisation des données de la sensation et donc de ce qui fonde la possibilité de la mesure. Le second, très court, mais dont la fin, renvoie directement à un article plus tardif sur la conception du calcul différentiel, discute des expérimentations physiques qui visent directement la conception classique de la mécanique. De 1893 à 1905, les étapes de cette solution se précisent touchant tous les points fondamentaux du puzzle qu'il doit composer : la nature du raisonnement mathématique, l'espace et la géométrie, la mesure du temps, jusqu'à la discussion sur calcul infinitésimal, tous les éléments de sa conception se mettent en place en vue d'une solution originale et très mal comprise par ses contemporains (sauf des rares exceptions) ${ }^{16}$, pour ne pas parler des interprètes ultérieures.

\section{LA PUISSANCE DE L'ESPRIT}

Disons d'emblée ce qu'est la solution de Poincaré au double défi de Boutroux et de Duhem et commençons par les deux premières sections de «La mesure du temps » d'abord publié en 1898 dans la RMM et ensuite reproduit presque à l'identique dans le deuxième chapitre de La Valeur de la science. L' article vise à montrer que la mesure du temps n'est que relative et que le temps en tant que grandeur mesurable, tout comme l'espace, est un cadre que nous imposons à la nature parce que nous le trouvons commode. Cependant ces deux premières sections introduisent une distinction qui me semble capitale, entre le temps de la conscience individuelle et le temps scientifique et physique. Ce dernier est le temps mesurable, dans lequel nous pouvons exprimer de combien un événement précède ou suit un autre. Le premier est le temps psychologique et qualitatif, dans lequel toutefois nous savons ordonner les événements de la conscience, dans un ordre qui suffit pour établir la simultanéité, l'antériorité ou la postériorité des sensations.

Tant que l'on ne sort pas du domaine de la conscience, la notion du temps est relativement claire. Non seulement nous distinguons sans peine la sensation présente du souvenir des sensations passées ou de la prévision des sensations futures ; mais nous savons parfaitement ce que nous voulons dire quand nous affirmons que, de deux phénomènes conscients dont nous avons conservé le souvenir, l'un a été antérieur à l'autre ; ou bien que, de deux phénomène conscients prévus, l'un sera antérieur à l'autre.

Quand nous disons que deux faits conscients sont simultanés, nous voulons dire qu'ils se pénètrent profondément l'un l'autre, de telle sorte que l'analyse ne peut les séparer sans les mutiler ${ }^{17}$.

Ce constat porte Poincaré à deux conclusions l'une et l'autre allant clairement dans le sens de l'affirmation de l'apriorité du temps psychologique qui n'a pas d'équivalent pour l'espace représentatif.

La première conclusion est que l'ordre temporel dans lequel nous rangeons les phénomènes conscients ne comporte aucun arbitraire. «Il nous est imposé et nous ne pouvons rien y changer $»$.

La seconde est que ce temps psychologique est une véritable forme pure, a priori, de la sensibilité, préexistante dans notre esprit à tout contenu ${ }^{18}$.

Il n'y a rien de comparable qui puisse être dit à propos de l'espace car comme Poincaré l'a déjà expliqué dans ses différentes interventions, non seulement la géométrie et construite par l'esprit à l'occasion de l'expérience, mais il n'y a aucun sens de l'espace qui nous ferait localiser nos sensations dans un espace représentatif tout fait qui s'imposerait à nous. Notre capacité de localisation ne se développe que dans l'interaction entre les capacités de notre esprit et des caractéristiques de notre environnement (la présence de corps rigides). Cette interaction permet l'association constante entre certaines sensations externes et certains

\footnotetext{
${ }^{16}$ Voir par exemple l'analyse subtile de Brunschvicg RMM 1913, t.25, pp.585-616.

${ }^{17}$ H. Poincaré, La Valeur de la science, op.cit., p.41.

18 « D'où vient » s'interroge-t-il « ce sentiment qu'entre deux instants quelconques il y a d'autres instants?

Nous classons nos souvenirs dans le temps, mais nous savons qu'il reste des cases vides. Comment cela serait-il possible si le temps n'était une forme préexistant dans notre esprit ? Comment saurions-nous qu'il y a des cases vides, si ces cases ne nous étaient révélées que par leur contenu? ». Idem, p. 42
} 
mouvements ou, plus précisément, certaines sensations musculaires internes liées à nos mouvements ou à leurs représentations.

Il y a donc une différence radicale entre le temps psychologique et l'espace psychologique ou représentatif, dont les conséquences sont tirées par Poincaré au troisième chapitre de $L a$ Valeur de la science. En effet Poincaré s'y demande :

[...] d'où vient à l'espace son caractère quantitatif? Il vient du rôle qui jouent dans sa genèse les séries de sensations musculaires. Ce sont des séries qui peuvent se répéter, et c'est de leur répétition que vient le nombre; c'est parce qu'elles peuvent se répéter indéfiniment que l'espace est infini. [...] Ainsi c'est la répétition qui a donné à l'espace ses caractères essentiels ; or la répétition suppose le temps ; c'est assez dire que le temps est antérieur logiquement à l'espace ${ }^{19}$.

Comme Poincaré l'a dit au chapitre précédent, la possibilité de découper notre flux expérientiel en éléments distincts est la conséquence des limites de notre mémoire, incapable de retenir le complexe des sensations présentes dans sa totalité. Une fois ainsi subjectivement étiquetés, les événements de la conscience, leur ordonnancement, est quelque chose qui s'impose à nous. Nous ne pouvons pas ne pas les ordonner, tout en sachant « qu'il reste des cases vides ». C'est ce savoir immédiat, intuitif de la possibilité indéfinie de la répétition qui, n'étant pas encore nombre, fonde la possibilité du nombre. A la différence de l'intuition pure $\mathrm{du}$ temps chez Kant, cette intuition demande que l'action volontaire s'introduise et accompagne par sa répétition l'intuition «qu'il reste des cases vides». Toutefois cette intuition poincaréenne peut jouer un rôle semblable à celle kantienne, puisqu'elle fait le lien entre ce qui est constitutif de l'expérience et ce qui est constitutif des mathématiques.

L'importance de cette déclaration de dépendance logique de l'espace au temps, que malheureusement on cherchera en vain dans d'autres écrits de Poincaré, réside dans le fait que la notion de répétition a été mise au cœur de la conception des mathématiques dans $L a$ Science et l'hypothèse à la fois dans l'explication du raisonnement mathématique et de la construction du continu. Poincaré y a en effet expliqué en quel sens la science nous permet de connaître les rapports entre les choses en organisant son argumentation en quatre parties : Le nombre et la grandeur, L'espace, La force et La nature. Cet ordre n'est pas fortuit, c'est un ordre des raisons. Il part de la connaissance pure de l'arithmétique, dépourvue de toute convention, pour arriver aux lois physiques, qui dépendent des données brutes de nos sens. Les domaines intermédiaires de la géométrie et de la physique générale, ou physique des principes, qui sont étudiés dans L'espace et La force, dépendent, par contre, de façon essentielle des conventions, la nature de chacune de ces sciences devant être analysée en fonction de l'usage qu'elles font des différents types d'hypothèses.

Or, les deux extrêmes opposés des mathématiques pures et de la physique expérimentale reposent sur un principe commun. Ce principe revient à généraliser à partir de la répétition d'un nombre fini de données élémentaires semblables les unes aux autres. Dans le cas des mathématiques, ces données proviennent de l'esprit, c'est celui-ci qui les construit. Dans le cas de la physique, ces données proviennent de l'observation de la nature.

De même qu'en physique nous concluons au caractère général d'une propriété après l'avoir observée sur un nombre fini de cas, en mathématiques, puisque l'esprit «se sait capable de concevoir la répétition indéfinie d'un même acte dès que cet acte est une fois possible » (p. 41), nous formons des propositions générales à partir de vérifications sur un nombre fini de cas.

Dans les mathématiques pures, l'homogénéité des données est garantie par l'esprit (il y a homogénéité des unités qui sont adjointes successivement) et l'intuition directe que

\footnotetext{
${ }^{19}$ Ibidem, p. 98 ; ce chapitre reprend un article publié toujours sur la $R M M$ en 1903 sous le titre «L'espace et ces trois dimensions ».
} 
l'esprit a de concevoir sa capacité de répéter un nombre indéfini de fois un même acte fonde la nécessité du raisonnement arithmétique. Cette caractéristique rend les véritables démonstrations arithmétiques irréductibles au principe analytique de non-contradiction (qui échoue devant l'infini), irréductible à l'expérience (elle aussi impuissante devant l'infini) et irréductible à n'importe quelle convention (car aucun choix n'est possible dans la généralisation du fini à l'infini).

Ce passage du particulier au général, reposant sur l'intuition de la répétition, fonde les mathématiques dans le sujet, lui confère son caractère à la fois synthétique et apodictique, lui confère son pouvoir créateur tout en dévoilant l'analogie frappante avec l'induction empirique. La structure des raisonnements inductifs en physique et en mathématique est la même, la différence étant qu'en physique, l'induction repose sur l'hypothèse d'un ordre général de l'univers, tandis qu'en mathématique ce même ordre ne dépend que de notre esprit.

Le rôle de l'intuition de la répétition indéfinie d'un acte sera a maintes reprises évoqué dans le reste de La Science et l'hypothèse, avec un renvoi explicite aux thèses énoncées dans le chapitre I : au chapitre II, à propos de la construction des nombres rationnels et réels par le procédé d'intercalation (p. 53); au chapitre IV (donc dans la deuxième partie L'Espace) à propos de l'axiome d'homogénéité des groupes, qui garantit l'applicabilité des géométrie à l'expérience (p. 88); au chapitre IX (dans la quatrième partie sur La Nature) à propos du processus d'intégration et de dérivation et du processus de généralisation des hypothèses physiques par le biais des mathématiques (pp. 171-2). Bien que l'intuition de la répétition indéfinie ne soit pas mentionnée dans la partie sur La Force, l'examen des principes de la mécanique se ramène toujours chez Poincaré à une discussion sur l'ordre des équations différentielles lesquelles sont également obtenues par la répétition indéfinie du même acte. Donc, l'analyse du premier chapitre de La Science et l'hypothèse rayonne donc sur l'ensemble du texte. Elle explique, pour le domaine le plus simple des mathématiques, en quel sens celles-ci sont fondées sur le sujet. Bref elle nous donne la clef de la possibilité de la connaissance.

\section{LES LIMITES ET LA SATISFACTION DE L'ESPRIT}

Et pourtant tout n'est pas réglé. Dans le cas de la physique, nous l'avons dit, l'induction empirique postule l'homogénéité de la matière, laquelle n'est jamais réelle, car il faudrait que les circonstances dans lesquelles se reproduit un phénomène soient toutes semblables, «mais cela n'arrivera jamais » (p. 26). L'homogénéité postulée de la matière est indispensable, mais seulement vraisemblable. Comment concilier cette affirmation avec la vérité des lois physiques?

Il convient de regarder de plus près l'article de 1893 sur le continu (repris comme nous l'avons dit comme chapitre II de La Science et l'hypothèse) dans la perspective d'un écrit plus tardif pour répondre à cette question.

L'intervention de Poincaré dans la RMM en 1893, intervention qui constitue la source de ce chapitre, évoque trois sortes de problèmes liés à la compréhension du continu et de son rapport au nombre: philosophique, mathématique, physique. Poincaré mentionne explicitement les problèmes liés à la compréhension philosophique du continu, comme d'une unité qui précède toutes ses parties et dont aucun élément ultime n'est discernable.

Concernant le problème mathématique, il cite les travaux de Tannery, de «l'école de Berlin » et de Dedekind, sur l'arithmétisation de l'analyse. Il relève explicitement la nécessité de comprendre l'origine du continu mathématique dans sa relation aux problèmes de la mesure des grandeurs. Dans le texte de 1893, il mentionne par ailleurs les travaux d'Helmholtz, en particulier Zahlen und Messen. Il convient d'examiner de près ce point de jonction des trois problèmes, où est proposée une solution originale qui n'est ni celle de Tannery ou de Dedekind, ni celle d'Helmholtz. Elle apparaît clairement si on rapproche le 
passage de l'introduction de La Science et l'hypothèse concernant le cadre de la grandeur, du chapitre II et de l'hommage à Cournot ${ }^{20}$. On comprend alors que la stratégie de Poincaré consiste à relier le problème mathématique aux problèmes physiques et philosophiques du continu, tout en indiquant que des solutions spécifiques doivent être formulées pour chacun d'eux.

La solution du problème mathématique du « continu » aboutit à une " discrétisation » de la continuité, les paradoxes qui pourraient surgir de cette situation étant résolus grâce à l'infinité des termes intercalés. De ce point de vue, pour Poincaré, il n'y a pas de différence théorique entre la nature dense, mais incomplète, des rationnels et la " complétude » des réels. La distinction tient seulement au fait que l'introduction des rationnels et celle des réels répondent à des paradoxes de type différent: respectivement ceux de la sensation et ceux de l'imagination. La réponse mathématique aux problèmes de fondements de l'analyse résulte de son arithmétisation et est jugée entièrement satisfaisante pour le mathématicien, ainsi que Poincaré le déclare ouvertement dans l'écrit de $1905^{21}$. C'est celle d'une construction sophistiquée et ouverte de tous les individus nécessaires pour satisfaire le besoin de l'entendement pour donner un cadre cohérent aux données de l'expérience. L'entendement ne peut raisonner que sur des individus discrets et identifiables. La sensation et l'imagination ne nous donnent accès qu'à des points nébuleux qui empiètent les uns sur les autres et qui, pour cette raison, ne sont pas susceptibles de supporter des conditions d'identification. Notre capacité de concevoir la répétition indéfinie de l'acte d'intercalation comble ce hiatus.

$\mathrm{La}$ résolution mathématique du problème du continu ne lève pas toutefois les problèmes, physique et philosophique, qui sont signalés, mais traités de manière seulement implicite en 1893 et donc dans le texte de 1902. L'article de 1905 reprend explicitement la question:

Est-ce à dire que l'étude des difficultés aujourd'hui vaincus, et des efforts qu'on a faits pour lutter contre elles, soit désormais dépourvue de tout intérêt ou n'ait plus qu'un intérêt historique? Il s'en faut de beaucoup ; il semble qu'en s'arithmétisant, en s'idéalisant pour ainsi dire, la mathématique s'éloignait de la nature et le philosophe peut toujours se demander si les procédés du calcul différentiel et intégral, aujourd'hui complètement justifiés au point de vue logique, peuvent être légitimement appliquées à la nature. Le continu que nous offre la nature et qui est en quelque sorte une unité est-il semblable au continu mathématique, tel que l'ont défini les plus récents géomètres, et qui n'est plus qu'une multiplicité d'éléments, en nombre infini, mais extérieurs les uns aux autres et pour ainsi dire logiquement discrets?» (Ibidem.p. 302)

Le problème physique du continu concerne la réelle adaptation de ce cadre mathématique aux données de l'expérience. Comment sommes-nous sûrs de ne pas tout fausser ? Comment sommes-nous sûrs que l'expérience ne nous amènera pas à abandonner ce cadre conventionnel? «Si l'on admet que les phénomènes naturels peuvent être représentés par des nombres et par conséquent par des fonctions mathématiques, les règles du calcul infinitésimal pourront être appliquées à ces fonctions et cela en toute rigueur [...] mais il reste précisément à savoir s'il existera une fonction mathématique susceptible de représenter les phénomènes avec une précision indéfinie» (Ibidem.). En effet, et c'est tout le point de l'article de 1893 , et du texte de $\mathrm{SH}$, l'observation nous donne, non pas un nombre, mais une sensation qui n'est justement pas exprimable elle-même par un nombre, étant indiscernable d'autres sensations trop voisines (Ibidem). Seulement dit Poincaré, nous admettons que cette imprécision n'appartient qu'aux sensations elles-mêmes, que «leur

\footnotetext{
${ }^{20}$ « Augustin Cournot et les principes du calcul infinitésimal » paru en 1905 dans la RMM, vol.13, pp293-306 et republié de façon posthume dans Dernières pensées pp. 300-324.

${ }^{21}$ «Ce sont les récents progrès de la théorie des fonctions qui ont fait disparaître les dernières difficultés [de compréhension des principes du calcul infinitésimal] ; le jour où on a défini le nombre incommensurable d'une façon satisfaisante, de façon à parfaire ce que l'on a appelé l'arithmétisation de l'analyse mathématique, les derniers voiles ont été levés, à tel point que nous avons aujourd'hui peine à comprendre ce qui a pu autrefois paraître obscur » Ibidem , p. 301.
} 
cause inconnue est susceptible d'être exactement représentée par un nombre » (Ibidem p. 303). Cette croyance, « ce postulat» (Ibidem. p.304) " sur lequel repose toute la science » (Ibidem p. 306), est soutenu par deux faits complémentaires: d'une part le constat de notre capacité à répéter nos expériences en diminuant la marge d'incertitude grâce à des instruments de plus en plus sophistiqués; d'autre part le fait que l'imprécision même de nos sens, grâce auxquels nous nous servons de ces instruments, nous garantit que cette imprécision ne sera jamais complètement éliminée :

Quelque multipliées et quelque précises que soient nos expériences, elles seront toujours entachées de certaines erreurs qu'on pourra réduire, mais non pas annuler. Il y aura donc toujours moyen de représenter les observations, quelles qu'elles soient, par des fonctions qui s'en écarteront moins que ne comporte l'incertitude des mesures et qui jouiront de la continuité, de la propriété d'avoir des dérivées, de toutes les propriétés des fonctions analytiques. Une fonction quelconque étant donnée, on peut toujours trouver une fonction analytique ${ }^{22}$ qui en diffère aussi peu que l'on veut. Ainsi le physicien peut toujours appliquer les règles du calcul infinitésimal sans craindre un démenti de l'expérience ». $D P$, p.307.

La résolution du problème physique du continu ouvre à son tour le problème philosophique de la nature de ces causes inconnues susceptibles d'être mesurées par des nombres : «C'est assez pour le physicien » ajoute Poincaré à la fin du passage cité plus haut dans le texte «ce n'est pas assez pour le philosophe [...]. » Ibidem p. 307. La question n'est plus tant ici d'ordre épistémologique, que d'ordre ontologique. Quelle conception pouvonsnous nous faire du rapport entre nous et le monde réel, au-delà de la forme mathématique que nous avons imposée à nos expériences? Quelle explication rationnelle peut donner satisfaction à l'esprit face à la conception de la loi impliquée par la résolution du problème physique du continu ? Tel est le problème philosophique du continu selon Poincaré. L'article de 1905 invoque à ce propos la conception des infiniment petits de Cournot, en ayant soin de rapporter sa distinction entre ordre rationnel et ordre logique. Le recours aux infinitésimaux ne peut pas résoudre les problèmes "logiques » des fondements de l'analyse, mais il peut donner satisfaction à l'esprit dans sa recherche d'explication. Poincaré livre sa propre conception à la fin de l'article. Les infiniment petits, qui sont la véritable raison des choses, ne sont pas des atomes, ni des infinis en acte (comme le soutient Cournot), mais de purs et perpétuels devenirs, « comme les infiniment petits leibniziens » (Ibidem p.318).

Pour nous, qui ne croyons pas à la possibilité de concevoir un monde extérieur indépendamment du sujet pensant, ce serait la solution la plus simple et la plus naturelle. La raison première fuirait toujours devant l'esprit qui la cherche sans jamais pouvoir l'atteindre, et ce serait l'infiniment petit leibnizien qui symboliserait le mieux cette fuite éternelle Ibidem p. $318^{23}$.

Les problèmes mathématique, physique et philosophique, du continu sont intimement reliés. Toutefois leur solution ne doit pas être recherchée dans une source unique.

\footnotetext{
22 Dans «L'œuvre mathématique de Weierstrass» Poincaré affirme à propos des fonctions analytiques «Aujourd'hui [...] on distingue deux domaines, l'un sans limites, l'autre plus restreint, mais mieux cultivé. Le premier est celui de la fonction en général, le second celui de la fonction analytique. Dans le premier, toutes les fantaisies sont permises et à chaque instant nos habitudes sont heurtées et nos associations d'idées rompues; nous y apprenons ainsi à nous défier de certains raisonnements par à peu près qui paraissaient convaincants à nos pères ; à nous abstenir de telles conclusions qui leur auraient paru légitimes. Dans le second, au contraire, ces conclusions sont permises ; mais nous savons pourquoi ; il a suffi de placer au début une bonne définition; et on a vu reparaître une rigoureuse logique. » («L'œuvre mathématique de Weierstrass », Acta Mathematica n.22 1898, pp.1-18) p. 5

${ }^{23}$ Il est intéressant de rapprocher ce passage de celui de la Théorie générale des fonctions (section 28, p. 81) de Du Bois-Reymond discutant des différentes options que l'Idéaliste a à sa disposition pour rendre compte du concept de limite. Après avoir mentionné « les analystes du siècle dernier » qui ont pris tout à fait au sérieux le concept d'infiniment petit en tant que quantité réelle extérieure au domaine commun des quantité, Du BoisReymond affirme « Plus tard des analystes, qui représentent une opinion intermédiaire, considèrent l'infiniment petit non pas sans doute comme une quantité ordinaire, mais au contraire comme une quantité qui s'écoule, en mouvement vers le zéro, saisie sur le point de s'évanouir, par conséquent comme quelque chose que nous avons appelé illimité en petitesse. »
} 
L'explication rationnelle qui convient à la raison (explication qui résout le problème philosophique) ne peut pas être utilisée par l'entendement dans son besoin de rigueur (problème mathématique), ni être appliquée à la sensation caractérisée par l'imprécision et la grossièreté de ses données (problème physique). Néanmoins, l'oubli de la liaison profonde qui caractérise ces trois aspects du problème, autant que la négligence de l'interdépendance entre ces trois solutions, ont des conséquences fâcheuses sur la pratique de la science dans son ensemble. 